\section{GENETICS}

\section{Carbohydrates weigh in on obesity genetics}

A recent study in humans has identified the first genetic link between body mass index (BMI) and carbohydrate metabolism (Nat. Genet. doi:10.1038/ng.2939).

Mario Falchi and his colleagues analyzed DNA arrays for common copy number variants (CNVs) in 651 subjects from 149 Swedish families that had pairs of siblings where one was obese and one was not. By linking the identified CNVs with gene expression data from adipose tissue (to ensure a phenotypic effect of the CNV) and BMI, the authors identified an inverse association between BMI and copy number of the region encoding the salivary amylase gene cluster ( $A M Y 1)$. This cluster encodes a protein called salivary amylase, which initiates carbohydrate digestion in the oral cavity. Quantitative PCR analyses of DNA copy number confirmed this association in another 3,600 European subjects, in which $A M Y 1$ copy number could account for $2.47 \%$ to $19.86 \%$ of the total genetic variation in obesity. Similar results were seen in 2,400 subjects from a Singaporean Chinese case control study, indicating that $A M Y 1$ copy number is not a European populationspecific genetic risk factor for obesity.

Amounts of plasma salivary amylase in 468 morbidly obese French subjects were linked to copy number and inversely associated with BMI. How these genetic alterations in $A M Y 1$ lead to obesity and how to therapeutically exploit this new link between digestive enzyme levels and obesity remain to be explored. - HS

\section{METASTASIS}

\section{Tumor cells unlock the endothelium}

At sites of metastasis, tumor cells influence resident stromal cells to enable metastatic colonization and growth. Weiying Zhou and colleagues now show that tumor cells use a microRNA, delivered by exosomes to the endothelium, to increase vascular permeability and metastasis (Cancer Cell 25, 501-515, 2014).

The researchers identified miR-105 in a screen for microRNAs in exosomes released by a human metastatic breast tumor cell line that could stimulate endothelial cell migration. By targeting the tight junction protein ZO-1, miR-105 delivered in exosomes to

IMMUNOLOGY

\title{
Gut lining eases inflammation
}

A molecule expressed on intestinal epithelial cells (IECs) that is involved in antigen presentation restrains intestinal inflammation during colitis by inducing anti-inflammatory molecules, according to a study in Nature (doi:10.1038/ nature13150).

CD1d is found on antigenpresenting cells and presents self and microbial lipid antigens, activating invariant natural killer (iNKT) cells and promoting intestinal

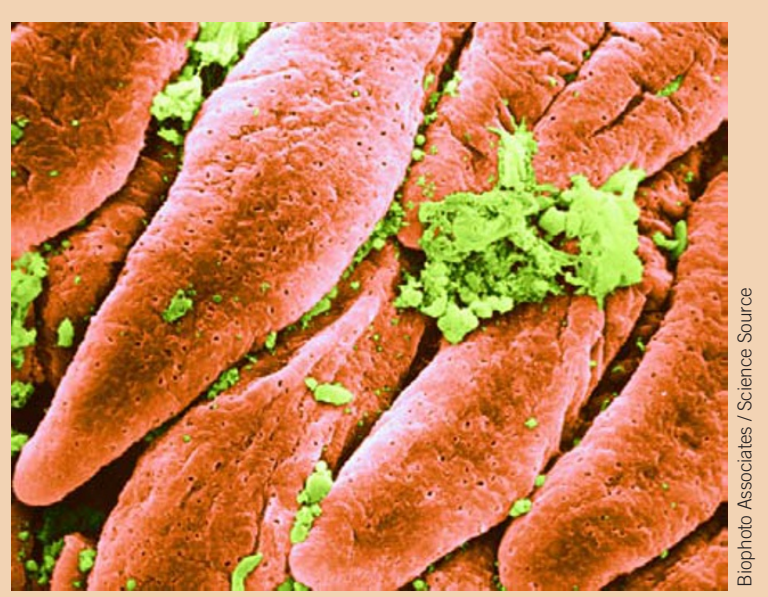
inflammation. The expression of $\mathrm{CD} 1 \mathrm{~d}$, however, is reduced on the intestinal epithelium in individuals with inflammatory bowel disease.

Richard S. Blumberg and colleagues now show that deletion of CD1d in IECs in an iNKT-cell dependent model of colitis worsens clinical outcome and increases mortality, whereas its absence in nonepithelial cells protected against colitis. Ligation of CD1d on the gut epithelium induces the expression of IL-10 and heat shock protein 110 (HSP110), which ameliorate inflammation. Loss of IL-10 in IECs also resulted in increased mortality and inflammation, suggesting that IL-10 from both IECs and bone marrow-derived cells is protective in models of colitis. These findings highlight a dichotomous role of CD1d in IECs and bone marrow-derived cells and the protective effect of IL-10 and HSP110 in intestinal inflammation. -KDS

endothelial cell monolayers increased both the permeability of the monolayers and the ability of tumor cells to invade across them. In mice, delivery of miR-105-containing exosomes or overexpression of miR-105 in metastatic breast cancer tumor cell xenografts decreased endothelial 20-1 expression and increased vascular permeability and metastasis, whereas miR-105 knockdown had the opposite effects.

In early-stage breast cancer patients, the authors detected miR-105 in exosomes in the blood and found that the levels of circulating or tumor miR-105 were inversely correlated with the level of tumor ZO-1 expression. The levels of circulating or tumor miR-105 were higher in patients who later developed distant metastases, suggesting the possible use of miR-105 as a predictive biomarker. $-M B$

\section{NEUROSCIENCE}

\section{Bringing back neuronal inhibition}

Some genetic versions of autism, such as Dravet syndrome, caused by mutations in the voltage-gated sodium channel gene Scnla, have been linked to reductions in inhibitory neurotransmission, but the extent to which this is also true for idiopathic forms of autism has been unclear. Now, Sung Han and colleagues show that dysfunctional inhibitory neurotransmission is responsible for social deficits in a nonsyndromic mouse model of autism (Neuron 81, 1282-1289, 2014).

The researchers carried out electrophysiological recordings from the hippocampus of BTBR mice, a model of idiopathic autism that displays many autistic-like behaviors such as dysfunctional social interactions and repetitive behaviors. They found reduced inhibitory neurotransmission in that brain region that could be restored with benzodiazepines, drugs that activate GABA receptors and are currently in clinical use to treat anxiety and epilepsy. The doses of benzodiazepines used to treat those disorders in humans can induce sedation as a side effect, but in these BTBR mice, even low, nonsedating doses could improve learning and social interactions. Conversely, treating two different lines of control mice that exhibit no autistic behaviors with drugs that 